\title{
Kinome Response Profile
}

National Cancer Institute

\section{Source}

National Cancer Institute. Kinome Response Profile. NCI Thesaurus. Code C107137.

A protein expression profile to define and quantitate the activity and drug responsiveness

of the expressed kinome. 